\title{
Macrophage activation syndrome associated with systemic juvenile idiopathic arthritis
}

\author{
Clovis Artur A. Silva, ${ }^{1}$ Carlos Henrique M. Silva, ${ }^{2}$ \\ Tereza Cristina M. V. Robazzi, 3 Ana Paola N. Lotito, 4 Alfredo Mendroni Junior, 5 \\ Cristina M. A. Jacob, ${ }^{6}$ Maria Helena B. Kiss ${ }^{7}$
}

\begin{abstract}
Objective: To describe the characteristics of macrophage activation syndrome associated with juvenile idiopathic arthritis.

Description: This is a retrospective study involving 462 patients with juvenile idiopathic arthritis. Seven (1.5\%) of those patients suffered from systemic onset juvenile idiopathic arthritis and developed macrophage activation syndrome. The median age of the juvenile idiopathic arthritis onset was 3 years and 10 months and the median duration of juvenile idiopathic arthritis before macrophage activation syndrome was 8 years and 4 months. All of them presented with fever, jaundice, hepatosplenomegaly, bleeding, pancytopenia, abnormal liver function tests and abnormal coagulation profile. Three cases presented associated infections and one patient developed macrophage activation syndrome two weeks after the administration of sulfasalazine. Three patients died and the macrophage hemophagocytosis was present in five. The treatment of macrophage activation syndrome included pulse therapy with methylprednisolone in all of them, cyclosporine A in three, plasma exchange in two and intravenous immunoglobulin in two.
\end{abstract}

Comments: Macrophage activation syndrome is a complication of the systemic onset juvenile idiopathic arthritis with a high morbidity and mortality rate.

J Pediatr (Rio J). 2004;80(6):517-22: Juvenile idiopathic arthritis, juvenile rheumatoid arthritis, macrophage activation syndrome, liver.

1. Ph.D. Head of the Pediatric Rheumatology Unit, Department of Pediatrics, School of Medicine, Universidade de São Paulo (USP), São Paulo, SP, Brazil.

2. Ph.D. Adjunct professor, Department of Pediatrics, School of Medicine, Universidade Federal de Uberlândia (UFU), Uberlândia, MG, Brazil.

3. Physician responsible for the Pediatric Rheumatology Unit, Hospital São Rafael, Salvador, BA, Brazil.

4. M.Sc., School of Medicine, Universidade de São Paulo (FMUSP). Assistant physician, Centro de Saúde Pinheiros, São Paulo, SP, Brazil.

5. Ph.D. Head of the Department of Apheresis, Fundação Pró-sangue, Hemocentro de São Paulo, São Paulo, SP, Brazil.

6. Ph.D. Head of the Immunology and Allergy Unit, Department of Pediatrics, School of Medicine, FMUSP, São Paulo, SP, Brazil.

7. Associate professor, School of Medicine, FMUSP, São Paulo, SP, Brazil.

Manuscript received Mar 03 2004, accepted for publication May 242004.

Suggested citation: Silva CA, Silva $\mathrm{CH}$, Robazzi TC, Lotito AP, Mendroni Junior AM, Jacob CM, et al. Macrophage activation syndrome associated with systemic juvenile idiopathic arthritis. J Pediatr (Rio J). 2004;80:517-22.

\section{Introduction}

The new, internationally adopted classification for childhood arthritis defines the term juvenile idiopathic arthritis (JIA), substituting juvenile rheumatoid arthritis (JRA), as chronic arthritis (persisting for more than 6 weeks) in one or more joints with onset before 16 years of age and unknown etiology. The diagnosis of JIA is one of exclusion and includes seven different groups: systemic arthritis, oligoarthritis, rheumatoid factor positive polyarthritis, rheumatoid factor negative polyarthritis, psoriatic arthritis, arthritis associated with enthesitis and undifferentiated arthritis. The systemic form of JIA is defined as the presence of arthritis in one or more joints associated with daily fever above $39^{\circ} \mathrm{C}$ for a minimum period of 15 days together with the presence of at least one of the following manifestations: rheumatoid exanthema, 
generalized adenomegaly, pericarditis, pleuritis, hepatomegaly and/or splenomegaly. ${ }^{1}$

The macrophage activation syndrome (MAS) is an entity with both familiar and acquired forms. The acquired forms can be triggered by infections, neoplasms, immunodeficiencies and rheumatic diseases. ${ }^{2-5}$ Macrophage activation syndrome associated with JIA is extremely rare. There are no reported prevalence rates and around 88 cases had been described by 2001.4

Macrophage activation syndrome associated with JIA was first described by Hadchouel et al. in 1985.6 This syndrome occurs primarily with the systemic form of JIA, and the average period of JIA prior to MAS onset is from 4.2 to 4.8 years. 6,7

Other names have been used in published literature for this syndrome when associated with JIA, such as: reactive hemophagocytic syndrome, 5,8 hemophagocytic lymphohistiocytosis ${ }^{2}$ or disseminated intravascular coagulation (DIVC) with liver failure. ${ }^{9}$

Patients with MAS present prolonged high fever, hepatomegaly and splenomegaly, bleeding, generalized adenomegaly, rash, jaundice, can develop acute liver failure, coma, DIVC and multiple organ failure. ${ }^{2-5}$ Anemia, leukopenia, thrombocytopenia, hypofibrinogenemia and slowed coagulation habitually occur in all patients. ${ }^{10,11}$ The presence of numerous macrophages in the bone marrow phagocytosing blood cells (hemophagocytosis), with no evidence of malignancy is characteristic of MAS. ${ }^{3,12}$

Macrophage activation syndrome can be triggered by viral infectious agents such as: varicella-zoster, ${ }^{13}$ hepatitis $A,{ }^{14}$ Epstein-Barr ${ }^{8}$ and coxsackie $B,{ }^{15}$ in addition to therapy with gold, acetylsalicylic acid and other nonsteroidal antiinflammatory drugs, ${ }^{6,12}$ methrotrexate, ${ }^{10}$ sulfasalazine and penicillamine. $^{7}$

The etiopathogenesis of MAS is unknown. The macrophage activation liberates proteases which activate plasminogen, leading to the formation of plasmin with fibrin degradation, triggering fibrinolysis and DIVC. ${ }^{3}$ Cytokine overproduction, particularly of interleukin 1, tumor necrosis factor and gamma-interferon, observed in children with familial hemophagocytic lymphohistiocytosis, can be responsible for abnormalities affecting the liver brain and blood in such children. ${ }^{16}$ Macrophage activation syndrome involves overproduction of cytokines originating from activated T lymphocytes and macrophages (IFN- $\gamma$, GM-CSF, IL-6, IL-1 and TNF $\alpha$ ), significant reductions in NK cells ${ }^{17}$ and perforin (the protein that controls the cytotoxic activity of NK cells and T lymphocytes). ${ }^{18}$

The objective of the present study was to describe the clinical characteristics, laboratory findings and therapies employed in children and adolescents with MAS associated with JIA.

\section{Case descriptions}

Clinical and laboratory protocols were used to retrospectively analyze the medical records of all 462 children and adolescents who had been diagnosed with JIA according to ILAR criteria (International League of Associations of Rheumatology) ${ }^{1}$ during the period between January 1983 and January 2002. The patients had all been treated at the Pediatric Rheumatology Unit at the FMUSP Pediatrics Department. The MAS diagnosis was defined as abrupt onset of pancytopenia associated with jaundice, elevated transaminases, direct bilirubin and coagulation rates $^{3}$ and/or from evidence of blood cell phagocytosis by macrophage proliferation. 4

The following clinical characteristics were analyzed: JIA onset and course of disease, the presence of recent infectious processes, characterization of liver involvement and medication-based treatment previously given. The course of the JIA prior to MAS onset was classed as either remissive (disease inactive for at least 2 years) ${ }^{19}$ or polycyclic (periods of disease activity and inactivity).

The following workup exams had been performed: complete blood test, erythrocyte sedimentation rate (ESR), aspartate transaminase (AST), alanine aminotransferase (ALT), prothrombin time (PT), thrombin time (TT), activated partial thromboplastin time (APTT), fibrinogen, factor $\mathrm{V}$, fibrin degeneration products (FDP), albumin, total bilirubin and fractions, triglycerides, myelogram and serum tests for Epstein-Barr virus, cytomegalovirus, rubella and hepatitis $A, B$ and $C$. All histological examinations performed at autopsy were evaluated by the same pathologist from the Pathological Anatomy Service at FMUSP. This research was approved by the Research and Ethics Commission at the FMUSP Hospital das Clínicas.

During the study period seven (1.5\%) cases of systemic JIA that developed MAS were monitored. These originated from a total of 462 JIA patients, 198 (43\%) of whom had the systemic form. Seven (1.5\%) patients developed MAS and all of them had had the systemic form of the disease. Table 1 lists the demographic characteristics of these patients. Five were male and five were white. The median for age at JIA onset was 3 years and 10 months and the median JIA duration prior to MAS onset was 8 years and 4 months. The period between onset of symptoms and MAS diagnosis varied from 2 to 30 days, with a median of 5 days. The cases in question occurred between 1993 and 1998.

Table 2 illustrates the workup exams and treatments for MAS associated with systemic JIA. All patients presented persistent high fevers (habitually above $39^{\circ} \mathrm{C}$, with a mean duration of 30 days) and acute liver failure with jaundice, hepatosplenomegaly (an average of $3 \mathrm{~cm}$ from the costal margin) and bleeding, in particular gingival bleeding and petechiae. In four cases JIA was reactivated with polyarthritis involving knees, ankles and wrists; and two presented rheumatoid rash concomitantly with the febrile episode. Two patients had been in clinical and laboratory remission from JIA, one of them for 1 year (case 1 ) and the other for 3 years (case 3 ).

With respect of previous infectious history, case 1 presented a co-infection of hepatitis $\mathrm{C}$ and cytomegalovirus with evidence of IgM antibodies for both infections, case 3 developed MAS 1 week after a hepatitis A outbreak at the 
patient's residence, confirmed by anti-VHA IgM serology. Case 6 developed MAS two weeks after chickenpox.

Hepatic enzymes were at elevated levels in all patients (mean AST was $800 \mathrm{U} / \mathrm{I}$ and ALT was $600 \mathrm{U} / \mathrm{I}$ ). All patients exhibited pancytopenia, elevated coagulation times and increased direct bilirubin (mean $11.7 \mathrm{mg} / \mathrm{dl}$ ). Four cases presented normal or reduced EST (less than $20 \mathrm{~mm}$ in the first hour). Acute renal insufficiency with elevated urea levels (above $60 \mathrm{mg} / \mathrm{dl}$ ) and creatinine (above $1.2 \mathrm{mg} / \mathrm{dl}$ ) was observed in four patients. Hypoalbuminemia (below 3 $\mathrm{g} / \mathrm{l}$ ) was found in three and hypertriglyceridemia (above 160 $\mathrm{mg} / \mathrm{dl}$ ) in six patients. In four cases the bone marrows showed macrophage proliferation with phagocytosis, particularly affecting erythrocytes (hemophagocytosis); case 7 exhibited bone marrow hypoplasia with no macrophage proliferation.

Prior to MAS, systemic JIA treatment had included nonsteroidal anti-inflammatory drugs (in particular acetylsalicylic acid and indomethacin), corticosteroids and disease-modifying antirheumatic drugs in all cases. The drugs that were being given at the time that MAS signs and symptoms appeared are listed in Table 2 . They had been being used for between 2 months and 4 years prior to MAS onset. It should be emphasized that only in case 5 did MAS clearly develop two weeks after the introduction of sulfasalazine.

Table 1 - Demographic characteristics of seven patients with macrophage activation syndrome (MAS) associated with juvenile idiopathic arthritis (JIA)

\begin{tabular}{|c|c|c|c|c|c|c|c|}
\hline Characteristic & 1 & 2 & 3 & 4 & 5 & 6 & 7 \\
\hline Sex & M & M & M & M & M & $\mathrm{F}$ & $\mathrm{F}$ \\
\hline Race & $\mathrm{C}$ & $\mathrm{C}$ & $\mathrm{C}$ & $\mathrm{C}$ & $\mathrm{C}$ & B & B \\
\hline Age at JIA onset & $3 y 1 \mathrm{~m}$ & $5 y 9 m$ & $3 y 7 m$ & $4 y 4 m$ & $2 y 2 m$ & $3 y 10 m$ & $7 y 3 m$ \\
\hline JIA duration prior to MAS onset & $5 y 4 m$ & $11 y$ & $6 y$ & $10 y 4 m$ & $8 y 4 m$ & $2 y 9 m$ & $12 y 8 m$ \\
\hline Period between onset of symptoms and MAS diagnosis & $10 d$ & $7 d$ & $30 d$ & $3 d$ & $2 d$ & $3 d$ & $5 d$ \\
\hline
\end{tabular}

$\mathrm{M}=$ male $; \mathrm{F}=$ female $; \mathrm{C}=$ Caucasian $; \mathrm{B}=$ black $; \mathrm{y}=$ years $; \mathrm{m}=$ months $; \mathrm{d}=$ days.

Table 2 - Complementary exams and treatments of seven patients with macrophage activation syndrome (MAS) associated with juvenile idiopathic arthritis (JIA)

\begin{tabular}{|c|c|c|c|c|c|c|c|}
\hline Exam & 1 & 2 & 3 & 4 & 5 & 6 & 7 \\
\hline $\mathrm{AST}>40 \mathrm{U} / \mathrm{I}$ & + & + & + & + & + & + & + \\
\hline$A L T>40 \mathrm{U} / \mathrm{I}$ & + & + & + & + & + & + & + \\
\hline $\mathrm{Hb}<10 \mathrm{~g} / \mathrm{dl}$ & + & + & + & + & + & + & + \\
\hline Leukocytes $<4,000 / \mathrm{mm}^{3}$ & + & + & + & + & + & + & + \\
\hline Platelets $<100,000 / \mathrm{mm}^{3}$ & + & + & + & + & + & + & + \\
\hline$\uparrow$ Direct bilirubin & + & + & + & + & + & + & + \\
\hline 个 PT, APTT and TT & + & + & + & + & + & + & + \\
\hline$\downarrow$ Factor I, V and FDP & + & NR & NR & + & + & + & NR \\
\hline Triglycerides > $160 \mathrm{mg} / \mathrm{dl}$ & + & + & + & NR & + & + & + \\
\hline Abnormal myelogram & - & - & + & + & + & + & + \\
\hline JIA treatment before MAS & $\begin{array}{l}\text { A, C, } \\
\text { I, P }\end{array}$ & $\begin{array}{l}\text { A, C, I, } \\
\text { G, P }\end{array}$ & $\begin{array}{c}\text { A, C, I, } \\
\text { N, P }\end{array}$ & $\begin{array}{l}\text { A, C, I, } \\
\text { Mt, G, P }\end{array}$ & $\begin{array}{c}\text { A,C, I, } \\
M t, G, P, S\end{array}$ & $\begin{array}{l}\text { A, C, I, } \\
\text { Mt, P }\end{array}$ & $\begin{array}{l}\text { A, C, I, } \\
\text { Ib, Mt, P }\end{array}$ \\
\hline $\begin{array}{l}\text { JIA treatment at the onset } \\
\text { of MAS symptoms }\end{array}$ & $\mathrm{I}, \mathrm{Mt}, \mathrm{P}$ & - & - & $\mathrm{A}, \mathrm{Mt}, \mathrm{P}$ & $I, P, S$ & $A, P$ & $A, P$ \\
\hline MAS treatment & $\mathrm{Cp}, \mathrm{M}$ & M & M & $\mathrm{Cp}, \mathrm{M}, \mathrm{PI}$ & $\mathrm{Cp}, \mathrm{IG}, \mathrm{M}$ & $\mathrm{M}, \mathrm{PI}$ & M, IG \\
\hline
\end{tabular}

AST = aspartate transaminase; $\mathrm{ALT}=$ alanine aminotransferase; $\mathrm{Hb}=$ hemoglobin; $\mathrm{PT}=$ prothrombin time; $\mathrm{APTT}=$ activated partial thromboplastin time; $\mathrm{TT}=$ thrombin time; FDP = fibrin degeneration products; $\mathrm{NR}=$ non registered; + = present; - = absent; $\mathrm{A}=$ acetylsalicylic acid; $\mathrm{C}=$ chloroquine; I = indomethacin; $\mathrm{P}$ = prednisone; $\mathrm{G}=$ gold salts; $\mathrm{N}=$ naproxen; $\mathrm{Mt}=$ methotrexate; $\mathrm{S}=$ sulfasalazine $; \mathrm{lb}=$ ibuprofen; $\mathrm{Cp}=$ cyclosporine; $\mathrm{M}=$ pulse therapy with methylprednisolone; $\mathrm{PI}=$ plasmapheresis; IG = intravenous gammaglobulin . 
The treatment for MAS initially included daily pulse therapy with methylprednisolone (30 mg/kg/day) in all cases, ranging from three to five pulse therapies per patient. Five patients required one or more treatments to control the disease (Table 2): two patients were given plasmapheresis (three sessions with 1.5 plasma volume exchange and intervals of 24 to 48 hours); two received intravenous gammaglobulin ( $2 \mathrm{~g} / \mathrm{kg} / \mathrm{single}$ dose) and three received intravenous cyclosporine $(2 \mathrm{mg} / \mathrm{kg} /$ day every 12 hours). Early diagnosis and introduction of pulse therapy with methylprednisolone and cyclosporine were associated with improved progress (disappearance of symptoms and normalization of leukocytes, platelets and coagulation times, in an average of 1 week).

All patients exhibited somnolence and sleep inversion. Three patients required admission to the intensive care unit due to coma, requiring mechanical ventilation and went on to die from multiple organ failure. The autopsies of these three patients showed, in case 1 , bleeding diathesis, splenitis, periportal fibrosis and hepatic steatosis; in case 2 bleeding diathesis, chronic pericarditis, splenitis, acute renal insufficiency and liver necrosis; there was no evidence of hemophagocytosis in the first two cases; in case 3 findings were, bleeding diathesis, massive liver necrosis (compatible with fulminating hepatitis) and macrophage proliferation in the lungs, with hemophagocytosis.

\section{Comments}

Around $10 \%$ of children with JIA present with the systemic form. ${ }^{20}$ At the Pediatric Rheumatology Service, which gives priority to caring for more serious and complex cases, the systemic form is the most common of the subtypes and accounts for $41 \%$ of the JIA cases. ${ }^{21}$

Evidence suggests that MAS etiopathogenesis is associated with a hereditary defect in immunoregulation, predisposing sufferers to a histiocytic proliferation in response to specific triggering agents such as viruses, 15,22 autoimmune diseases, ${ }^{6,22-28}$ immunodeficiencies (such as during the accelerated phase of the Chédiak-Higashi syndrome $)^{3}$ and drugs. 7,10

In the majority of studies of JIA, the criteria for MAS diagnosis are not defined. Ravelli ${ }^{4}$ performed a retrospective study of 88 patients with JIA (72 reported in published literature and 16 new Italian cases). The variables that offered greatest sensitivity and specificity (both above $0.75)$ were : ferritin $>10,000 \mathrm{ng} / \mathrm{ml}(1.0 ; 1.0)$, triglycerides $>160 \mathrm{mg} / \mathrm{dl}(0.9 ; 1.0)$, TGO > $40 \mathrm{UI} / \mathrm{ml}(0.93 ; 0.97)$, fibrinogen < $250 \mathrm{mg} / \mathrm{dl}(0.85 ; 1.0)$, TGP > $40 \mathrm{UI} / \mathrm{ml}(0.87$; $0.93)$, thrombocytopenia $<150,000 / \mathrm{mm}^{3}(0.76 ; 1.0)$, bone marrow with macrophage proliferation and hemophagocytosis $(0.75 ; 1.0)$, hepatomegaly $(0.76 ; 0.86)$ and splenomegaly $(0.77 ; 0.82)$.

Macrophage activation syndrome associated with JIA occurs primarily in children, ${ }^{2-5}$ but can also affect young adults who present JIA onset during childhood and after disease durations of 2 to 12 years, $6,8,14$ or, rarely, can occur with Still's disease in adults. 23,26
In the current survey, the median period of JIA duration prior to development was 8 years, characterizing a long duration of an immunosuppressive disease, with possibilities for infections and the need to employ a number of different therapeutic regimens with multiple side effects. The mean period of JIA duration before MAS onset described in the relevant literature varies from 4.2 to 4.8 years. 6,7 Schwartz et al. ${ }^{9}$ described a case of MAS with onset 17 years after the appearance of systemic JIA.

A previous infectious history associated with MAS was observed in three of our cases. Among patients with systemic JIA, the association of MAS with infectious agents has been described, on average one to two weeks after the onset of infection by the hepatitis $A$ virus, ${ }^{14}$ the varicella-zoster virus, ${ }^{13}$ coxsackie $B$ virus ${ }^{15}$ and the Epstein-Barr virus. ${ }^{8,26}$ Other associations such as: Pneumocystis carinii, salmonelosis ${ }^{27}$ cytomegalovirus, rubella, human immunodeficiency virus, bacterial diseases such as tuberculosis, leishmaniasis, brucellosis and syphilis; neoplasms and prolonged soluble lipid use have all been described. ${ }^{2}$

A number of different medications have been described as being possible triggers for MAS, in particular nonsteroidal anti-inflammatory drugs (acetylsalicylic acid and indomethacin), gold salts triggering the syndrome after the second weekly dose, ${ }^{6,12}$ methotrexate, ${ }^{10,27}$ penicillamine ${ }^{7}$ and sulfasalazine.7,27 All of our patients had been given a variety of different medications prior to the appearance of MAS. One of our cases presented MAS two weeks after the introduction of sulfasalazine.

Macrophages or histiocytes are seen actively phagocytosing erythrocytes in between 40 and $82 \% .4,5$ In the present study five patients presented macrophage proliferation with hemophagocytosis; four in bone marrow aspirate and one in the lungs at autopsy. Recently, hyperferritinemia has been associated with MAS positive patients in up to $100 \%$ of cases. ${ }^{4}$ Our last case was diagnosed in 1988 when this exam was not yet used routinely.

Macrophage activation syndrome must be differentiated from the systemic activity of the JIA syndrome itself. Fever is persistent in MAS, in contrast with systemic JIA where it occurs once or twice a day. ${ }^{3,7}$ The characteristic blood test results indicating systemic JIA activity will show leukocytosis in $80 \%$ and thrombocytosis in $70 \%$ of cases, in contrast with MAS which progresses with leukopenia and thrombocytopenia. ${ }^{21}$

All of our cases presented just one episode of MAS, with regression of jaundice and bleeding, on average one week after treatment was begun. Stéphan et al. ${ }^{5}$ describe two MAS relapses in $4 \%$ and three in $13 \%$ of their patients.

In order to treat the hemophagocytic syndrome, initially all NSAIDs, disease-modifying antirheumatic drugs and/or immunosuppressors should be withdrawn. Corticosteroids are the drugs of choice, intravenously, particularly pulse therapy with methylprednisolone.6,24 The second drug currently indicated in the literature is cyclosporine $A$, particularly for patients who are not responsive to 
corticosteroids. $3,7,24,25$ Other therapeutic modalities that can be employed are: intravenous gammaglobulin, cyclophosphamide, plasmapheresis ${ }^{12}$ and etanercepte. ${ }^{11}$

Three of these cases had fatal outcomes. All deaths were the result of liver failure and bleeding diathesis. These three patients all exhibited a prolonged time between onset of symptoms and seeking our Service, delaying MAS diagnosis.

Fatal outcomes are the result of 2 to $4 \%$ of children with JIA, particularly associated with the systemic form of the disease. ${ }^{21}$ Death rates vary from $1 \%$ in the United States to $4.2 \%$ in Europe, primarily due to amyloidosis. 29 Bernstein $^{30}$ describes deaths due to liver failure in $20 \%$ of patients. Boone ${ }^{31}$ describes death rates of $17 \%$ of a total of 1,674 patients with JIA from liver failure, generally from unknown causes, being occasionally due to viral hepatitis and the use of gold salts, believing that these deaths were in fact due to MAS. Deaths from MAS associated with systemic JIA, in studies with more than five cases, occurred in 11 to $30 \%$ of cases. ${ }^{5-7}$

We conclude that MAS is a complication of the systemic form of JIA with elevated morbidity and mortality. The presence of acute liver failure and pancytopenia in patients with JIA should alert health professionals to a diagnosis of MAS. Early recognition, differentiation from systemic activity of the systemic JIA itself and the rapid introduction of aggressive treatment contribute to better prognosis.

\section{Acknowledgements}

We would like to thank Dr. Fabíola del Carlo Bernardi for evaluating the autopsies of the patients in this study and Professor Dr. Gilda Porta, Dr. Bernadete de Lourdes Liphaus, Dr. Jorge David Aivazoglou Carneiro, Dr. Lúcia Maria M. A. Campos and Dr Nélson Hidekazu Tatfui, for their help in the diagnosis, treatment and follow-up of these patients.

\section{References}

1. Petty RE, Southwood TR, Manners P, Baum J, Glass DN, Goldenberg J, et al. International League of Associations for Rheumatology classification of juvenile idiopathic arthritis: second revision, Edmonton; 2001. J Rheumatol. 2004;31:390-2.

2. Henter JI, Elinder G, Öst A. Diagnostic guidelines for hemophagocytic lymphohistiocytosis. The FHL Study Group of the Histiocyte Society. Semin Oncol. 1991;18:29-33.

3. Grom AA, Passo M. Macrophage activation syndrome in systemic juvenile rheumatoid arthritis. J Pediat. 1996;129:630-2.

4. Ravelli A. Macrophage activation syndrome. Curr Opin Rheumatol. 2002; 14:548-52.
5. Stéphan JL, Koné-Paut I, Galambrun C, Mouy R, Bader-Meunier $B$, Prieur AM. Reactive haemophagocytic syndrome in children with inflammatory disorders. A retrospective study of 24 patients. Rheumatol. 2001;40:1285-92.

6. Hadchouel M, Prieur AM, Griscelli C. Acute hemorrhagic, hepatic, and neurologic manifestations in juvenile rheumatoid arthritis: possible relationship to drugs or infection. J Pediat. 1985; 106:561-6.

7. Sawhney S, Woo P, Murray KJ. Macrophage activation syndrome: a potentially fatal complication of rheumatic disorders. Arch Dis Child. 2001;85:421-6.

8. Fishman D, Rooney M, Woo P. Successful management of reactive haemophagocytic syndrome in systemic-onset juvenile chronic arthritis. Br J Rheumatol. 1995; 34:888-93.

9. Schwartz $D$, Averbuch $M$, Pines A, Kornovsky $R$, Levo $Y$. Disseminated intravascular coagulation with renal and liver damage as the predominant manifestations of recurrent relapses in systemic juvenile rheumatoid arthritis. Ann Rheum Dis. 1992;51:347-9.

10. Ravelli A, Caria MC, Buratti S, Mulattia C, Temporini F, Martini A. Methotrexate as a possible trigger of macrophage activation syndrome in systemic juvenile idiopathic arthritis. J Rheumatol. 2001;28:865-7.

11. Prahalad S, Bove KE, Dickens D, Lovell DJ, Grom AA. Etanercept in the treatment of macrophage activation syndrome. J Rheumatol. 2001;28:2120-4.

12. Bray $V$, Singleton JS. Disseminated intravascular coagulation in still's disease. Semin Arthritis Rheum. 1994;24:222-9.

13. Morris JA, Adamson AR, Holt PJL, Davson J. Still's disease and the virus associated haemophagocytic syndrome. Ann Rheum Dis. $1985 ; 44: 349-53$.

14. Mcpeake JR, Hirst WJR, Brind AM, Williams R. Hepatitis A causing a second episode of virus associated haemophagocytic lymphohistiocytosis in a patient with Still's disease. J Med Virol. 1993;39:173-5.

15. Heaton DC, Moller PW. Still's disease associated with Coxsackie infection and haemophagocytic syndrome. Ann Reum Dis. 1985;44:341-4.

16. Henter JI, Elinger G, Söder $O$, Hansson M, Andersson B, Andersson U. Hypercytokinemia in familial hemophagocytic lymphohistiocytosis. Blood. 1991;78:2918-22.

17. Grom AA, Villanueva J, Lee S, Goldmuntz EA, Passo $M H$, Filipovich A. Natural killer cell dysfunction in patients with systemic-onset juvenile rheumatoid arthritis and macrophage activation syndrome. J Pediatr. 2003;142:292-6.

18. Wulffraat NM, Rijkers GT, Elst E, Brooimans R, Kuis W. Reduced perforin expression in systemic juvenile idiopathic arthritis is restored by autologous stem-cell transplantation. Rheumatology. 2003;42:375-9

19. Hanson $V$, Kornreich $H$, Bernstein $B$, King KK, Singsen BH. Prognosis of juvenile rheumatoid arthritis. Arthritis Rheum (Supplement). 1977;20:279-84.

20. Cassidy JT, Petty RE. Juvenile rheumatoid arthritis. In: Cassidy JT, Petty RE, editors. Textbook of Pediatric Rheumatology. 4th ed. Philadelphia: WB Saunders Company; 2001. p. 218-321.

21. Silva CAA, Kiss MHB. Manifestações extra-articulares iniciais em 80 pacientes com artrite reumatóide juvenil (ARJ) forma sistêmica. Pediatria (São Paulo). 1998;20:83-92.

22. Loy TS, Diaz-Arias AA, Perry MC. Familial erythrophagocytic lymphohistiocytosis. Sem Oncol. 1991;18:34-9.

23. Dino O, Provenzano G, Giannuoli G, Sciarrino E, Pouyet M, Pagliaro L. Fulminant hepatic failure in adult onset Still's disease. J. Rhematol. 1996;23:784-5.

24. Mouy R, Stephan JL, Pillet P, Haddad E, Hubert P, Prieur AM. Efficacy of cyclosporine $A$ in the treatment of macrophage activation syndrome in juvenile arthritis: report of five cases. $]$ Pediatr. 1996;129:750-4.

25. Ravelli A, Benedetti F, Viola S, Martini A. Macrophage activation syndrome in systemic juvenile rheumatoid arthritis successfully treated with cyclosporine. J Pediatr. 1996;128:275-8.

26. Stern A, Riley R, Buckley L. Worsening of macrophage activation syndrome in a patient with adult onset Still's disease after initiation of etanercept therapy. J Clin Rheumatol. 2001;7:252-6.

27. Sibilia J, Javier RM, Albert A, Cazenave JP, Kuntz JL. Pancytopenia secondary to hemophagocytic syndrome in rheumatoid arthritis treated with methotrexate and sulfasalazine. J Rheumatol. 1998;25:1218-20. 
28. Sekigawa I, Suzuki J, Nawata M, Ikeda K, Koike M, Iida N, et al. Hemophagocytosis in autoimmune disease. Clin Exp Rheumatol. 2001;19:333-8.

29. Baum J, Gutowska G. Death in juvenile rheumatoid arthritis. Arthritis Rheum (Supplement). 1977;20:253-5.

30. Bernstein B. Death in juvenile rheumatoid arthritis. Arthritis Rheum (Supplement). 1977;20:256.

31. Boone JE. Hepatic disease and mortality in juvenile rheumatoid arthritis. Arthritis Rheum (Supplement). 1977;20:257-9.
Corresponding author:

Clovis Artur Almeida Silva

Rua Senador César Lacerda Vergueiro, 494/82, Vila Madalena

CEP 05435-010 - São Paulo, SP

Brazil

Phone: +55 (11) 3069.8675

Fax: +55 (11) 3069.8503

E-mail: clovisaas@icr.hcnet.usp.br 